(2) Open Access Full Text Article

\title{
Development of a tin oxide carrier with mesoporous structure for improving the dissolution rate and oral relative bioavailability of fenofibrate
}

This article was published in the following Dove Press journal:

Drug Design, Development and Therapy

\author{
Andi Bai \\ Chao Wu \\ Xuan Liu \\ Huiling Lv \\ Xiaoyan Xu \\ Yue Cao \\ Wenjing Shang \\ Lili Hu \\ Ying Liu \\ Department of Pharmaceutics, \\ School of Pharmacy, Jinzhou Medical \\ University, Jinzhou I21000, Liaoning, \\ People's Republic of China
}

\begin{abstract}
Background: Biopharmaceutics classification system class II drugs have low solubility, which limits their extent and speed of absorption after oral administration. Over the years, mesoporous materials have been widely used to increase the dissolution rate and oral relative bioavailability of poorly water-soluble drugs.

Objectives: In order to improve the dissolution rate and increase oral relative bioavailability of the poorly water-soluble drugs, a tin oxide carrier (MSn) with a mesoporous structure was successfully synthesized.
\end{abstract}

Methods: In this study, MSn was synthesized using mesoporous silica material (SBA-15) as the template. Fenofibrate (FNB) was adsorbed into the channels of MSn by an adsorption method. Characterizations of the pure FNB, MSn, physical mixture of the drug and MSn (PM; 1:1) and FNB-loaded MSn (FNB-MSn) samples were carried out by the scanning electron microscopy (SEM), transmission electron microscopy (TEM), $\mathrm{N}_{2}$ adsorption/desorption, powder X-ray diffractometer (PXRD), differential scanning calorimetry (DSC) and Fourier transform infrared (FT-IR) spectroscopy. Cytotoxicity assay (MTT) was used to evaluate the cytotoxicity of MSn. In vitro dissolution studies were performed to investigate the dissolution rate of FNB-MSn. In vivo pharmacokinetic studies were used to investigate the changes of plasma drug concentrations of FNB-MSn tablets and commercial FNB tablets in rabbits.

Results: Detailed characterization showed that FNB in the channels of MSn was present in an amorphous state. The in vitro release tests demonstrated that MSn with a good biocompatibility could effectively enhance the dissolution rate of FNB. Pharmacokinetic results indicated that MSn significantly increased the oral relative bioavailability of FNB.

Conclusion: MSn can be regarded as a promising carrier for an oral drug delivery system.

Keywords: tin oxide, mesoporous material, fenofibrate, dissolution rate, oral relative bioavailability

\section{Introduction}

Over the past decades, a poorly water-soluble drug is an enormous challenge in pharmaceutical preparations. Many poorly soluble drugs have low oral bioavailability due to poor water solubility. To improve the dissolution rate, various formulation strategies such as reduced crystallite sizes, ${ }^{1}$ solid microcrystalline dispersion, ${ }^{2}$ solid dispersion $^{3}$ and nanocrystal ${ }^{4}$ have been adopted and have achieved considerable success. In the recent past years, many pharmaceutical studies have been performed to solve this problem using mesoporous materials, such as mesoporous carbon
Correspondence: Chao Wu

Department of Pharmaceutics, School of Pharmacy, Jinzhou Medical University, 40 Songpo Road, Linghe, Jinzhou 121000 , Liaoning, People's Republic of China Tel +864l64673439

Email zifengas123@I63.com
Drug Design, Development and Therapy 2018:12 2129-2138

2129

Dovepress ff in 0

http://dx.doi.org/10.2147/DDDTS166989 (c) (1) (-) 2018 Bai et al. This work is published and licensed by Dove Medical Press Limited. The full terms of this license are available at https://www.dovepress.com/terms.php hereby accept the Terms. Non-commercial uses of the work are permitted without any further permission from Dove Medical Press Limited, provided the work is properly attributed. For permission for commercial use of this work, please see paragraphs 4.2 and 5 of our Terms (https://www.dovepress.com/terms.php). 
materials ${ }^{5-8}$ and mesoporous silicon materials..$^{9-11}$ The advantage of mesoporous materials is that nanoscale channels can restrict poorly water-soluble drugs from being present in an amorphous state to increase its dissolution rate and prevent the drug from recrystallization. ${ }^{12}$ He et al ${ }^{13}$ used mesoporous silica nanoparticles (MSNs) to raise the solubility of paclitaxel. Zhang et al ${ }^{14}$ utilized highly ordered mesoporous carbon (HMC) matrices to increase simvastatin release and enhance its oral bioavailability. Yang et $\mathrm{al}^{15}$ designed amorphous mesoporous magnesium carbonate and successfully stabilized tolfenamic acid and rimonabant in their amorphous forms to enhance their bioavailability. Geszke-Moritz and Moritz ${ }^{16}$ exploited four APTES-modified mesoporous silicas as matrix to improve the dissolution rate of diflunisal.

Currently, an increasing number of researchers are focused on mesoporous metal oxides such as mesoporous $\mathrm{ZnO}$ nanospheres ${ }^{17}$ and mesoporous alumina ${ }^{18}$ as a drug carrier to improve the dissolution rate of insoluble drugs. These metal oxide materials have the common advantages of mesoporous materials such as high surface area and large pore volume. ${ }^{19}$ Recently, tin oxide has received widespread attention in the field of pharmaceutical research as a result of its application in monitoring the drug release by fluorescent signal. ${ }^{20}$ Tin oxide is an n-type semiconductor with chemical stability, high specific surface area and good biocompatibility, has optical properties in the visible spectral range and is extensively used in the fields of transparent conductors, ${ }^{21}$ gas sensors, ${ }^{22}$ biosensors,${ }^{23}$ catalysis, ${ }^{24}$ energy storage $^{25}$ and medicinal applications. ${ }^{26,27}$ However, so far, there have been no previous reports of the oral administration of mesoporous tin oxide-based formulations. In this study, we prepared a novel tin oxide carrier with a mesoporous structure to improve the dissolution rate of a poorly watersoluble drug. Fenofibrate (FNB), a typical class II drug defined by the biopharmaceutics classification system (BCS), was selected as a model drug. FNB is virtually insoluble in water and physiological fluids. ${ }^{28}$ We utilized the mesoporous structure of MSn to increase the dissolution rate of FNB, and through in vitro drug release and in vivo pharmacokinetic study we verified that MSn was suitable as a carrier for the oral administration of poorly soluble drugs.

\section{Materials and methods}

\section{Materials}

FNB with purity $>99 \%$ was provided by the Dalian Meilun Company (Dalian, China). Tin (II) chloride dihydrate $\left(\mathrm{SnCl}_{2} \cdot 2 \mathrm{H}_{2} \mathrm{O}\right)$ was obtained from Sinopharm Chemical Reagent Co., Ltd. (Shanghai, China). Poly(ethylene glycol)block-poly(propylene glycol)-block-poly(ethylene glycol),
P123, was purchased from Sigma-Aldrich Co. (St Louis, MO, USA). Tetraethyl orthosilicate (TEOS) was supplied by Tianjin Bodi Chemical Holding Co., Ltd. (Tianjin, China). Dichloromethane was obtained from Tianjin Guangfu Fine Chemical Co., Ltd. (Tianjin, People's Republic of China). Hydrochloric acid, glacial acetic acid, methyl alcohol, hydrofluoric acid and anhydrous ethanol were purchased from Tianjin Yongsheng Fine Chemical Co., Ltd. (Tianjin, People's Republic of China). The human colon adenocarcinoma Caco-2 cell lines were purchased from National Platform of Experimental Cell Resources (Beijing, People's Republic of China). Deionized water was used in all experiments.

\section{Synthesis of SBA-I5 and MSn Step I}

In a typical synthesis of SBA- $15,{ }^{29} 2.0 \mathrm{~g}$ of nonionic triblock copolymer (Pluronic P123) was added to a homogeneous solution comprised of distilled water $(15 \mathrm{~g})$ and $60 \mathrm{~g}$ of $\mathrm{HCl}$ solution $(2 \mathrm{M})$ under vigorous stirring at $40^{\circ} \mathrm{C}$. Then, $4.25 \mathrm{~g}$ of TEOS was added to the abovementioned solution at $40^{\circ} \mathrm{C}$ under stirring for $24 \mathrm{~h}$. Next, the mixture was stirred at $100^{\circ} \mathrm{C}$ for 3 days. The precipitate obtained was filtered and dried at room temperature. The solid products were calcined in air at $500^{\circ} \mathrm{C}$ for $6 \mathrm{~h}$ to remove the organic template. The product obtained was SBA-15.

\section{Step 2}

MSn was prepared via an adsorption method using SBA-15 as the template. ${ }^{30,31} \mathrm{SnCl}_{2} \cdot 2 \mathrm{H}_{2} \mathrm{O}$ was dissolved in $10 \mathrm{~mL}$ of ethanol to obtain a solution $(3 \mathrm{M})$. Then, a certain amount of SBA-15 sample was added to the abovementioned solution. The mixture was stirred at room temperature for $24 \mathrm{~h}$ after sonicating for $1 \mathrm{~h}$. The precipitate obtained by centrifugation was dried at room temperature and calcined at $550^{\circ} \mathrm{C}$ in air for $5 \mathrm{~h}$. Finally, the template was removed by using $20 \%$ hydrofluoric acid (HF) solution at room temperature to obtain MSn.

\section{Drug loading}

FNB was loaded into MSn using a slightly modified version of the method reported by Xu et al. ${ }^{32}$ Briefly, FNB was dissolved in dichloromethane and mixed with MSn in a mass ratio of $1 / 1$. The mixture was stirred for $5 \mathrm{~h}$ in a closed container. After centrifugation, the supernatant was removed and the loaded sample (FNB-MSn) was dried in a vacuum. The loading capacity was determined by ultraviolet (UV) spectrophotometry at a wavelength of $286 \mathrm{~nm}$. The drug-loading content (DLC) of MSn was calculated by the following equation: DLC $(\%)=$ amount of drug present in MSn/total amount of FNB-MSn $\times 100 \%$. 


\section{Characterization of MSn and FNB-MSn}

The morphology and structure of the SBA-15 and MSn were analyzed by scanning electron microscopy (SEM; JEOL JSM-7001F, operated at $20 \mathrm{kV}$; JEOL, Tokyo, Japan) and transmission electron microscopy (TEM; Tecnai G2F30; operated at $200 \mathrm{kV}$; FEI, The Netherlands). Nitrogen adsorption-desorption isotherms were measured by using a surface area and pore size analyzer (SA3100; Beckman Coulter, Brea, CA, USA), and the Brunauer-Emmett-Teller (BET) and the Barrett-Joiner-Halenda (BJH) methods were used to calculate surface area, pore volume and diameter distribution of MSn according to the isotherms. The physical state of FNB in MSn was investigated using a powder X-ray diffractometer (PXRD; Rigaku Geigerflex XRD, Co., Tokyo, Japan; $\mathrm{Cu} \mathrm{K} \alpha$ radiation, $30 \mathrm{kV}$ and $30 \mathrm{~mA}$ Philips). Measurements were taken using a step size of $0.02^{\circ}$ at a scan rate of $4 \% \mathrm{~min}$. A differential scanning calorimeter (DSC; DSC-60; Shimadzu, Kyoto, Japan) was utilized to examine the drug crystallinity. DSC analysis was carried out using a heating rate of $10^{\circ} \mathrm{C} / \mathrm{min}$ from $50^{\circ} \mathrm{C}$ to $300^{\circ} \mathrm{C}$ under nitrogen at a flow rate of $150 \mathrm{~mL} / \mathrm{min}$. The Fourier transform infrared (FTIR) spectra of the samples were recorded using an FTIR spectrometer (Bruker IFS 55; Bruker Corporation, Billerica, MA, USA) with the aid of $\mathrm{KBr}$ pellets in the wavenumber range of $400-4,000 \mathrm{~cm}^{-1}$.

\section{Cytotoxicity assay (MTT)}

MTT assay was performed to evaluate the cytotoxicity of MSn. The Caco-2 cells were cultured for $24 \mathrm{~h}$ at a density of $1 \times 10^{5}$ cells/well in 96-well polystyrene-coated plates at $37^{\circ} \mathrm{C}$ in a $\mathrm{CO}_{2}$ incubator. MSn with a concentration of $250 \mu \mathrm{g} / \mathrm{mL}, 100 \mu \mathrm{g} / \mathrm{mL}, 50 \mu \mathrm{g} / \mathrm{mL}, 20 \mu \mathrm{g} / \mathrm{mL}, 10 \mu \mathrm{g} / \mathrm{mL}$ and $5 \mu \mathrm{g} / \mathrm{mL}$ was added into 96 -well polystyrene-coated plates. After $24 \mathrm{~h}$ of incubation, the MTT reagent $(20 \mu \mathrm{L}$, $5 \mathrm{mg} / \mathrm{mL}$ ) was added to each well and then further incubated for $4 \mathrm{~h}$. Dimethylsulfoxide (DMSO; $150 \mu \mathrm{L}$ ) was added, and the plate was vibrated for 10 min under dark conditions. A microplate reader (Biotek, Winooski, VT, USA) was used to record absorbance at $492 \mathrm{~nm}$. All experiments were performed in triplicate $(n=3)$.

\section{In vitro dissolution}

In vitro dissolution studies were performed according to the USP II paddle method using a dissolution instrument (ZRS-8G; Tianjin Xintianguang, Tianjin, People's Republic of China). The dissolution medium was an aqueous solution containing $1 \%$ of sodium dodecyl sulfate. ${ }^{33}$ The temperature was maintained at $37^{\circ} \mathrm{C} \pm 0.5^{\circ} \mathrm{C}$, and the paddle speed was $100 \mathrm{rpm}$. The sample specification was $100 \mathrm{mg}$, and the medium volume was $1,000 \mathrm{~mL}$. Then, $5 \mathrm{~mL}$ of samples was taken at $5 \mathrm{~min}, 10 \mathrm{~min}, 15 \mathrm{~min}, 20 \mathrm{~min}, 30 \mathrm{~min}, 45 \mathrm{~min}$ and $60 \mathrm{~min}$ and passed through a $0.45 \mu \mathrm{m}$ microporous membrane filter. The same amount of fresh dissolution medium was simultaneously added to the dissolution cup to maintain a constant total volume. The concentration of FNB was measured by UV spectrophotometry (UV-2000; Unico, Franksville, WI, USA) at a wavelength of $286 \mathrm{~nm}$.

\section{Preparation of FNB-MSn tablets}

FNB-MSn tablets were prepared by powder direct compression technology. The mixtures of FNB-MSn powders and the excipients were ground in a mortar and passed through an 80 mesh sieve. Finally, FNB-MSn tablets were obtained using a TDP single-punch tableting machine (TDP-1.5; Shanghai Wangqun, Shanghai, People's Republic of China) equipped with flat punches with a diameter of $8 \mathrm{~mm}$. In addition, we also measured the hardness, friability and disintegration time of the tablets.

\section{In vivo pharmacokinetic study}

Animals and dosing

Rabbits $(2.5 \pm 0.5 \mathrm{~kg})$ were supplied by the Jinzhou Medical University Laboratory Animal Center (Jinzhou, People's Republic of China). All experimental procedures, used in the current study, were performed according to the relevant international regulations of the Guide for the Care and Use of Laboratory Animals and were approved by the Jinzhou Medical University Laboratory Animal Ethics Committee for animal experiments. Six rabbits were randomly divided into two groups. All rabbits were fasted for $12 \mathrm{~h}$ before the experiment but allowed free access to water throughout the study. One group was given FNB-MSn tablets, and the other group was given commercial FNB tablets. Each rabbit was given the same preparation containing $100 \mathrm{mg}$ drug. Blood samples $(3 \mathrm{~mL})$ were withdrawn from the ear vein and placed in heparinized tubes at $5 \mathrm{~min}, 15 \mathrm{~min}, 30 \mathrm{~min}, 45 \mathrm{~min}, 1 \mathrm{~h}$, 1.5 h, 2 h, 4 h, 6 h, 8 h, 10 h, 12 h, 24 h and 36 h after drug administration by oral gavage. The blood samples were centrifuged at 4,250 rpm for $10 \mathrm{~min}$ using a high-speed centrifuge (TG22-WS; Shanghai Danding, Shanghai, People's Republic of China), and the obtained plasma samples were stored at $-20^{\circ} \mathrm{C}$ until used for analysis.

\section{Determination of FNB in plasma}

For analysis, plasma samples (each $400 \mu \mathrm{L}$ ) were transferred to $10 \mathrm{~mL}$ centrifuge tubes, followed by the addition of $20 \mu \mathrm{L}$ internal standard solution (methanolic indomethacin solution, $200 \mu \mathrm{g} / \mathrm{mL}$ ), $20 \mu \mathrm{L}$ methanol and $400 \mu \mathrm{L} \mathrm{HCl}$ aqueous solution $(1 \mathrm{~mol} / \mathrm{L})$. After vortex mixing for $3 \mathrm{~min}, 3 \mathrm{~mL}$ 
diethyl ether was added, and the solution was vortexed for a further $10 \mathrm{~min}$. Each sample was centrifuged at 4,250 rpm for $10 \mathrm{~min}$, and the supernatant was dried using a centrifugal drying machine. The residue was dissolved in $50 \mu \mathrm{L}$ mobile phase. Then, each sample was vortexed for $5 \mathrm{~min}$ and centrifuged at $12,750 \mathrm{rpm}$ for $10 \mathrm{~min}$, and $20 \mu \mathrm{L}$ supernatant was subjected to high-performance liquid chromatography (HPLC; L-2400; Hitachi Ltd., Tokyo, Japan) for analysis. The mobile phase was a mixture of methanol and $0.1 \%$ glacial acetic acid (75:25, vol/vol). The flow rate was $1.0 \mathrm{~mL} / \mathrm{min}$, and the UV detection wavelength was set at $286 \mathrm{~nm} .{ }^{34}$ The retention times of FNB and indomethacin were $10 \mathrm{~min}$ and 12 min, respectively. Analysis was carried out using an Agilent 5 TC-C18 column (Shandong Ruide, Shandong, People's Republic of China) $(250 \times 4.6 \mathrm{~mm})$.

\section{Statistical analyses}

All the pharmacokinetic data on FNB were analyzed by the pharmacokinetic analysis software DAS 2.0 (Mathematical Pharmacology Professional Committee of China, Shanghai, People's Republic of China). Several pharmacokinetic parameters, including the maximum peak concentration of the drug in plasma $\left(\mathrm{C}_{\max }\right)$, the time to reach the maximum concentration $\left(\mathrm{T}_{\max }\right)$ and the area under the curve (AUC), were obtained directly from the recorded results.

\section{Results and discussion} Preparation and characterization of MSn

The preparation process for MSn is illustrated in Scheme 1. First, the SBA-15 was synthesized by using the block copolymer surfactant P123 as the template agent. The P123 was removed by calcination to generate the mesochannels. ${ }^{35}$ Then, $\mathrm{SnCl}_{2} \cdot 2 \mathrm{H}_{2} \mathrm{O}$ was successfully incorporated into the mesochannels of SBA-15 (SBA-15-SnCl $\mathrm{S}_{2}$ ) using an adsorption method. Subsequently, the SBA-15- $\mathrm{SnCl}_{2}$ was calcined in air, and then the SBA-15 was removed using 20\% HF to obtain MSn. As shown in Figure 1A, the SEM image of SBA-15 as the template exhibited a regular rod-type morphology with a diameter of $\sim 0.5 \mu \mathrm{m}$ and a relatively uniform length of $3.0 \mu \mathrm{m}$. As we could see from the TEM image
(Figure 1B), SBA-15 showed a uniform and well-ordered mesoporous structure, and the average pore size of SBA-15 was $\sim 10 \mathrm{~nm}$. As shown in Figure 1C, SEM images of the replicated MSn clearly exhibited overall particle morphologies and sizes of MSn very similar to SBA-15. In addition, the TEM image, as shown in Figure 1D, clearly displayed the highly ordered and well-distinguishable mesostructures of MSn, and the pore size of MSn was the same as the pore size of SBA-15. These results confirmed that the MSn was successfully replicated from the SBA-15. Thus, MSn had the structural advantages, such as ordered pore structure, high surface area, high pore volume and high drug-loading capacity, as drug carriers such as SBA-15. ${ }^{36,37}$ Nanoscale channels of MSn could restrict the drug to be present in an amorphous state rather than the crystalline state. Therefore, the dissolution rate of drugs could be significantly improved. ${ }^{38}$ All the above mentioned structural characteristics indicated that synthetic MSn was suitable as an oral drug delivery carrier the same as other mesoporous materials. ${ }^{39,40}$

\section{Nanostructures and degree of drug loading}

The $\mathrm{N}_{2}$ adsorption/desorption isotherms and pore size distributions of MSn and FNB-MSn are shown in Figure 2A and B. Figure $2 \mathrm{~A}$ shows that MSn exhibited typical type IV isotherms according to the International Union of Pure and Applied Chemistry classification with a clear hysteresis loop, indicating the properties of typical mesoporous materials. ${ }^{41}$ Details of the textural properties of MSn and FNB-MSn are summarized in Table 1. MSn had a relatively high specific surface area $\left(\mathrm{S}_{\mathrm{BET}}\right)$ of $51.98 \mathrm{~m}^{2} / \mathrm{g}$, a relatively large total pore volume $\left(\mathrm{V}_{\mathrm{t}}\right)$ of $0.21 \mathrm{~mL} / \mathrm{g}$ and a pore diameter $\left(\mathrm{D}_{\text {влH }}\right)$ of $10.87 \mathrm{~nm}$. After FNB loading, the $\mathrm{S}_{\text {BET }}$, $\mathrm{V}_{\mathrm{t}}$ and $\mathrm{D}_{\text {ВлH }}$ of FNB-MSn were $17.54 \mathrm{~m}^{2} / \mathrm{g}, 0.08 \mathrm{~mL} / \mathrm{g}$ and $8.20 \mathrm{~nm}$, respectively. The $\mathrm{S}_{\mathrm{BET}}, \mathrm{V}_{\mathrm{t}}$ and $\mathrm{D}_{\text {ВगН }}$ of FNB-MSn were remarkably reduced compared with MSn, illustrating that FNB was successfully loaded into the MSn. In addition, drug loading also confirmed this explanation. Drug loading of MSn was up to $39.14 \%$, which was attributed to a large pore volume and the high specific surface area of MSn.

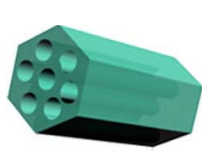

SBA-15
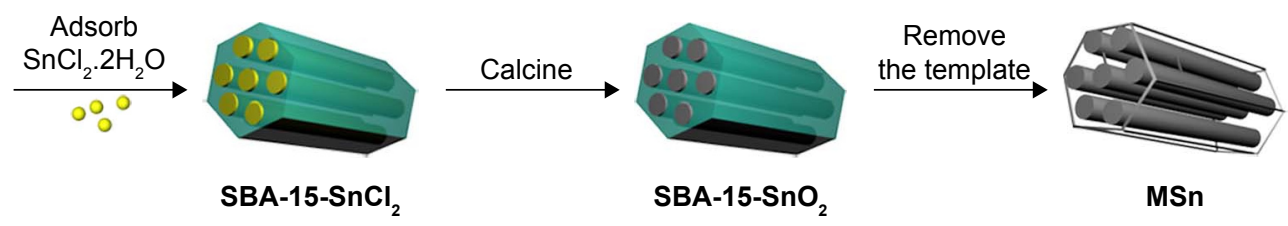

Scheme I Schematic illustration of the synthesis of MSn using SBA-I5 as templates. 

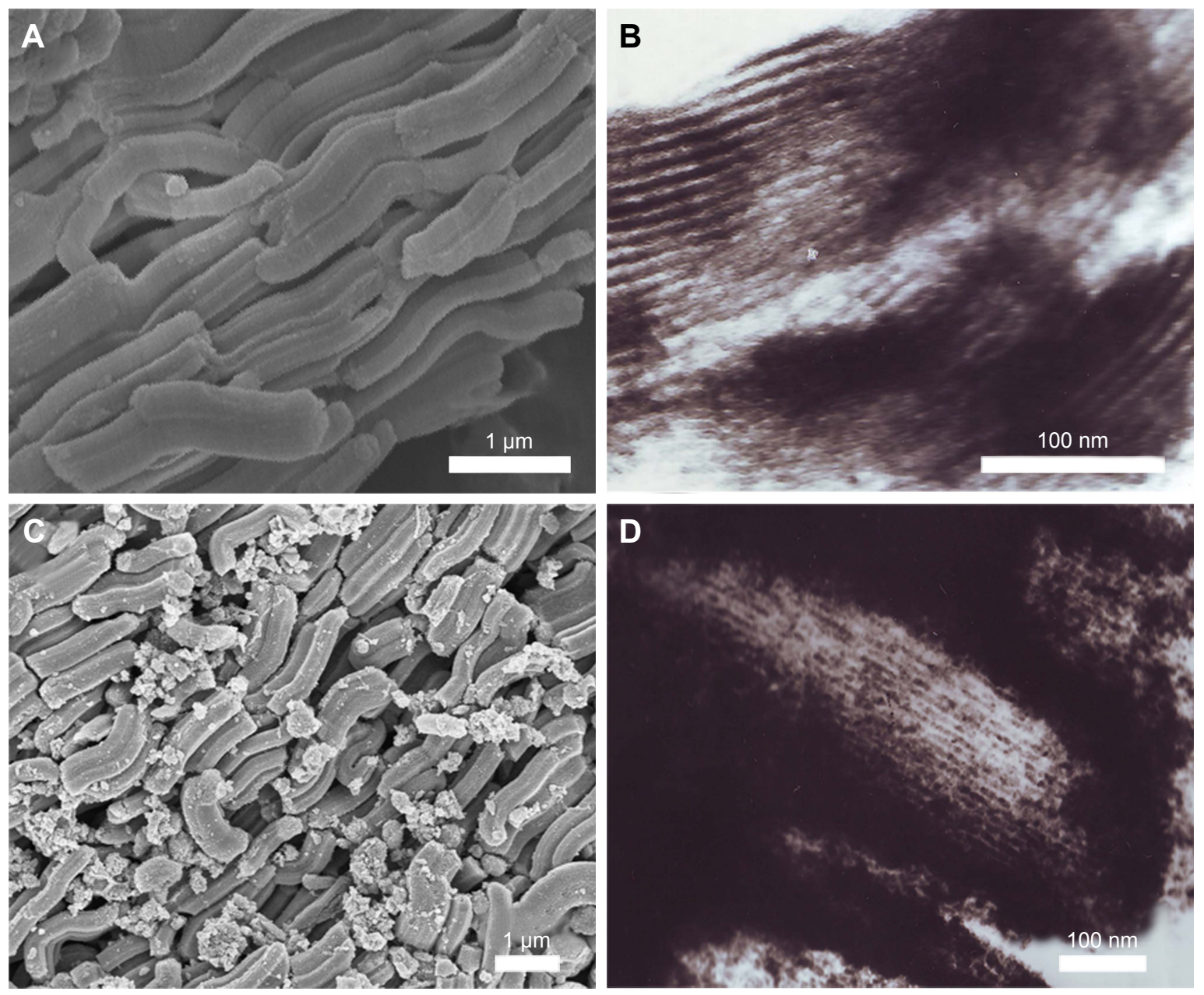

Figure I The SEM images of (A) SBA-I5 and (C) MSn. The TEM images of (B) SBA-15 and (D) MSn. Abbreviations: SEM, scanning electron microscopy; TEM, transmission electron microscopy.

A

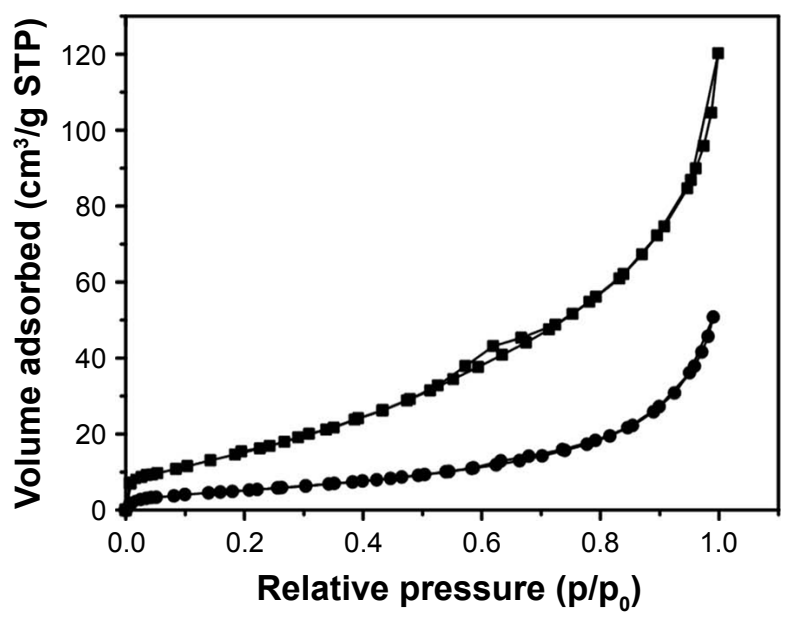

B

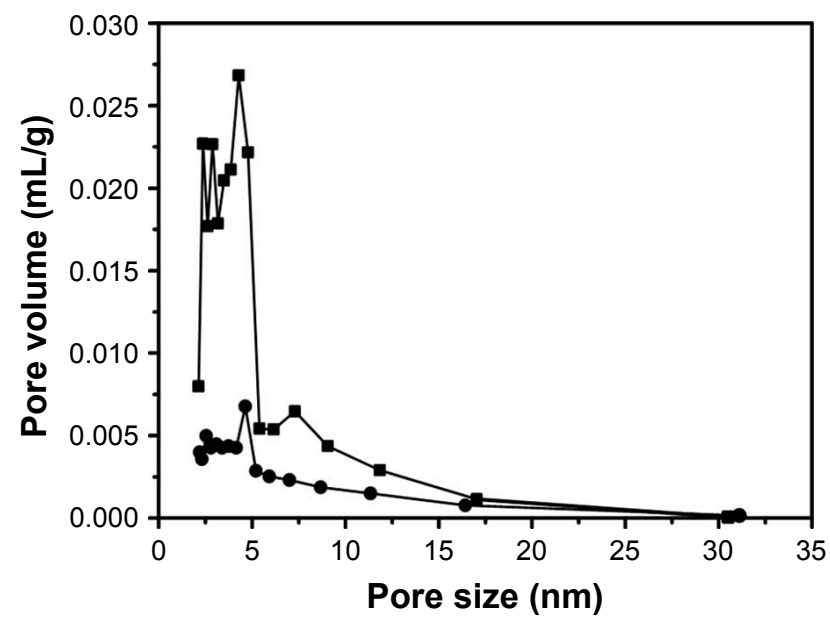

$\rightarrow-M S n \rightarrow F N B-M S n$

Figure $2 \mathrm{~N}_{2}$ adsorption-desorption isotherms (A) and pore size distributions (B) of MSn and FNB-MSn. Abbreviations: FNB, fenofibrate; STP, standard temperature and pressure. 
Table I Detailed textural properties of MSn and FNB-MSn obtained by $\mathrm{N}_{2}$ adsorption measurements

\begin{tabular}{llll}
\hline Samples & $\mathbf{S}_{\mathrm{BET}}\left(\mathrm{m}^{2} / \mathrm{g}\right)$ & $\mathbf{V}_{\mathrm{t}}(\mathrm{mL} / \mathrm{g})$ & $\mathbf{D}_{\mathrm{BJH}}(\mathrm{nm})$ \\
\hline MSn & 51.98 & 0.21 & 10.87 \\
FNB-MSn & 17.54 & 0.08 & 8.20 \\
\hline
\end{tabular}

Notes: $D_{B, H}$ pore diameter; $S_{B E T}$, specific surface area.

Abbreviations: BET, Brunauer-Emmet-Teller; BJH, Barrett-Joiner-Halenda; FNB, fenofibrate.

\section{PXRD and DSC characterization}

PXRD analysis was carried out to investigate the physical state of the FNB in MSn. The PXRD patterns of pure FNB, MSn, physical mixture of the drug and MSn (PM; 1:1) and FNB-MSn samples are shown in Figure 3. The diffraction pattern of pure FNB was highly crystalline as confirmed by a series of peaks. The characteristic diffraction peaks were at $20.98^{\circ}, 22.4^{\circ}, 24.84^{\circ}$ and $36.94^{\circ}$. The main diffraction peaks of MSn were distinctive at $26.7^{\circ}, 34.02^{\circ}, 36.82^{\circ}, 51.94^{\circ}$ and $54.78^{\circ}$, and these peaks can be well indexed to the (110), (101), (200), (211) and (220) planes of tetragonal $\mathrm{SnO}_{2}$, which was consistent with the previous literature. ${ }^{42}$ For the physical mixture, the main diffraction peaks were attributed to the superimposed peaks of pure FNB and MSn. However, no diffraction peak characteristic of pure FNB was observed in the FNB-MSn. This result indicated that the crystallinity of the drug is significantly restricted due to the mesoporous structure. FNB adsorbed in the pore channels of MSn was in an amorphous state, which was the main factor for enhancing the dissolution rate.

DSC was used to investigate the crystalline/amorphous state of the drugs in the carrier. ${ }^{43}$ The DSC curves of pure FNB, MSn, PM and FNB-MSn samples are shown in Figure 4 . The DSC curve of pure FNB displayed a single

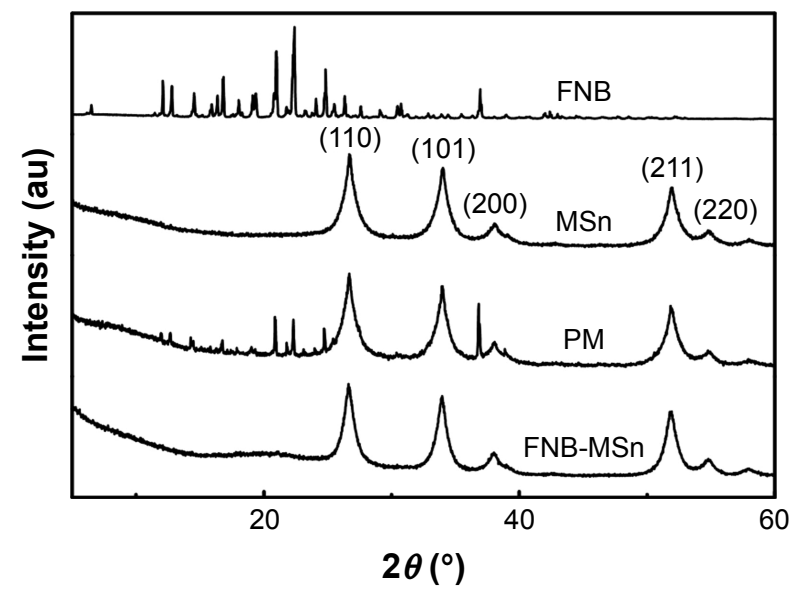

Figure 3 PXRD profiles of pure FNB, MSn, PM and FNB-MSn samples. Abbreviations: FNB, fenofibrate; PXRD, powder X-ray diffractometer; PM, physical mixture.

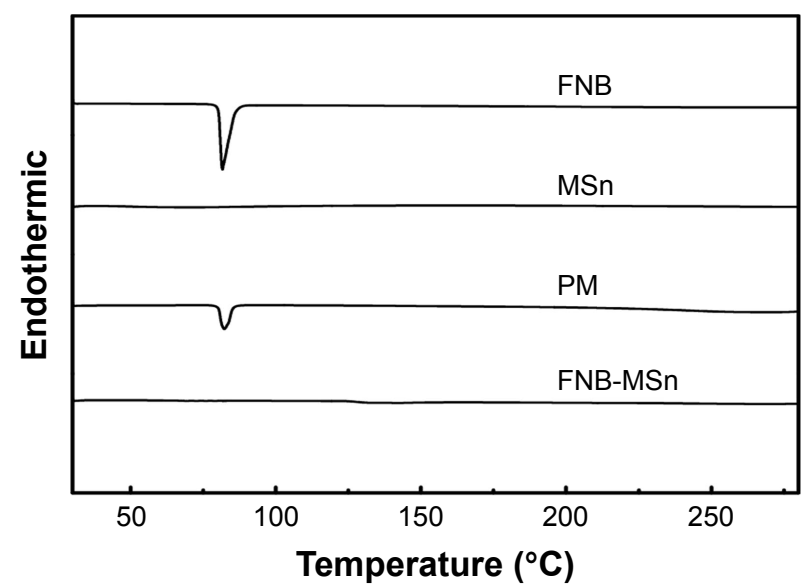

Figure 4 DSC thermograms for pure FNB, MSn, PM and FNB-MSn samples. Abbreviations: DSC, differential scanning calorimeter; FNB, fenofibrate; PM, physical mixture.

sharp endothermic peak at $83^{\circ} \mathrm{C}$, corresponding to its intrinsic melting point. The DSC thermogram of MSn did not exhibit any characteristic melting peak. The PM showed a slightly narrower melting peak than pure $\mathrm{FNB}$ at $83^{\circ} \mathrm{C}$, attributed to the melting of FNB, which demonstrated that MSn did not change the physical state of FNB in the PM. In contrast, for FNB-MSn, the phase transition temperature disappeared at $83^{\circ} \mathrm{C}$ relative to the melting of crystalline $\mathrm{FNB}$, which indicated that FNB was present in an amorphous state in the MSn. Consequently, the DSC further confirmed the results of PXRD characterization.

\section{FTIR characterization}

The FTIR spectra were used to identify possible chemical interactions between FNB and MSn. The FTIR spectra of pure FNB, PM, MSn and FNB-MSn are shown in Figure 5. The pure FNB showed characteristic bands at 2,983.88 $\mathrm{cm}^{-1}$,

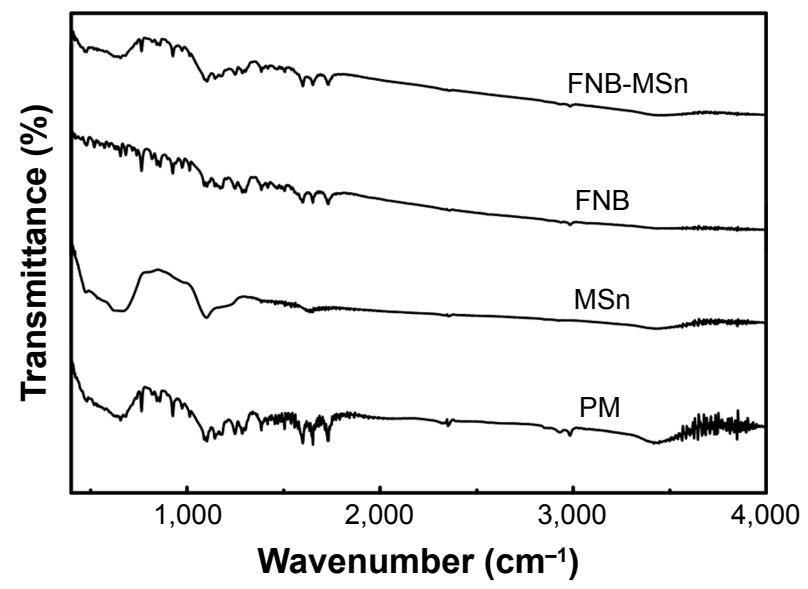

Figure 5 FTIR spectra of pure FNB, PM, MSn and FNB-MSn samples. Abbreviations: FNB, fenofibrate; FTIR, Fourier transform infrared. 
1,730.15 $\mathrm{cm}^{-1}$ and 1,651.07 $\mathrm{cm}^{-1} .44$ As shown in Figure 5, the FTIR spectrum of MSn displayed a hydroxyl stretching peak at 3,000-3,700 $\mathrm{cm}^{-1}$, which illustrated that MSn had a hydrophilic surface with abundant hydrophilic hydroxyl groups ${ }^{45}$ significantly increasing the contact of the FNB with water and reducing the diffusion resistance, which enhanced the dissolution of the FNB. The FTIR spectra of PM exhibited no modulation or new peaks at the characteristic peak position, indicating that there was no interaction between FNB and MSn. The characteristic peaks of PM were consistent with the FTIR spectra of FNB-MSn, again demonstrating that no interaction occurred between the FNB and MSn. These results indicated that the adsorption of FNB in MSn was a physical adsorption.

\section{Cytotoxicity assay}

MTT assay was used to determine the cell survival and growth rates. The principle was that the mitochondrial succinate-tetrazolium reductase system could transform the exogenous MTT to a purple-colored crystalline formazan in living cells. ${ }^{46}$ The amount of formazan generated was proportional to the number of viable cells..$^{47}$ To evaluate the biological safety of MSn, the results of the MTT assay are shown in Figure 6. The MSn showed a good biological safety on Caco-2 cells at all test concentrations. Even at the high concentration of $250 \mu \mathrm{g} / \mathrm{mL}$, the cell viability was higher than $90.53 \%$ after $48 \mathrm{~h}$ incubation. The results demonstrated that MSn had better biocompatibility as a carrier for drug delivery.

\section{In vitro dissolution of FNB-MSn powders}

As shown in Figure 7, compared with the pure FNB, the FNB-MSn exhibited a significantly faster rate of drug dissolution. The FNB-MSn displayed a burst dissolution of 53\%

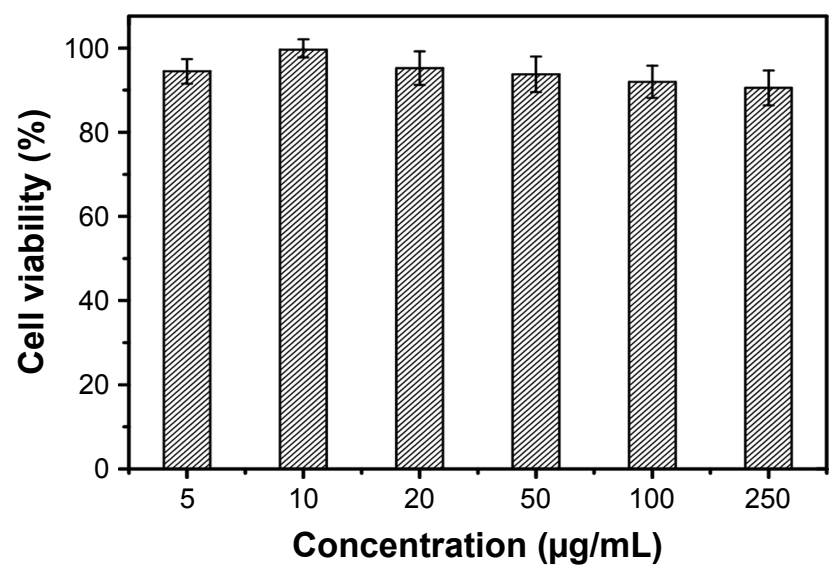

Figure 6 Cell viability of MSn at different concentrations $(n=3)$.

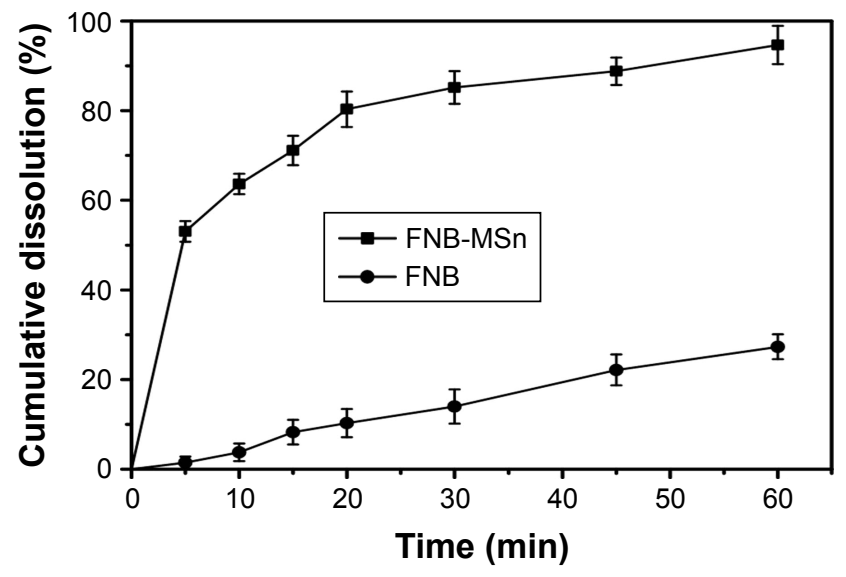

Figure 7 In vitro drug cumulative dissolution percentage patterns of pure FNB and FNB-MSn powders.

Abbreviation: FNB, fenofibrate.

within 5 min compared with only $1.45 \%$ for pure FNB dissolved during the same period. In addition, it took $1 \mathrm{~h}$ for pure FNB to dissolve $27 \%$ of drug, whereas $<5$ min was required for the FNB-MSn. At 1 h, 94\% of FNB from FNB-MSn was released. Owing to the space limitation effect, MSn nanopores inhibited the crystallinity of FNB, which resulted in the reduction in the size of the FNB particles. Therefore, FNB was present in an amorphous state in the mesoporous structure of MSn. Moreover, the specific surface area of the drug particles was increased. According to the Noyes-Whitney equation, the dissolution rate was proportional to the specific surface area of the drug particles. ${ }^{48}$ Therefore, MSn could significantly increase the dissolution rate of FNB, the major reason for improving the dissolution rate. These results confirmed that MSn was well suited as an oral drug carrier.

\section{Evaluation of FNB-MSn tablets}

The main factors affecting drug release from FNB-MSn tablets were adhesives, disintegrants, and fillers. Hence, we utilized a three-factor orthogonal experiment to explore the impact of these factors on drug release behavior and eventually got an optimal formulation (Table 2). As shown in Figure 8, the dissolution rate of FNB-MSn tablets showed

Table 2 Composition of FNB-MSn tablets

\begin{tabular}{lll}
\hline & Ingredients & $\begin{array}{l}\text { Formulation } \\
(\mathbf{m g})\end{array}$ \\
\hline Carrier & FNB-MSn & 255 \\
Adjuvant & Lactose & 80 \\
& PVP & 2 \\
& CMC-Na & 20 \\
& Magnesium stearate & 2 \\
\hline
\end{tabular}

Abbreviations: CMC-Na, sodium carboxyl methyl cellulose; FNB, fenofibrate; PVP, polyvinyl pyrrolidone. 


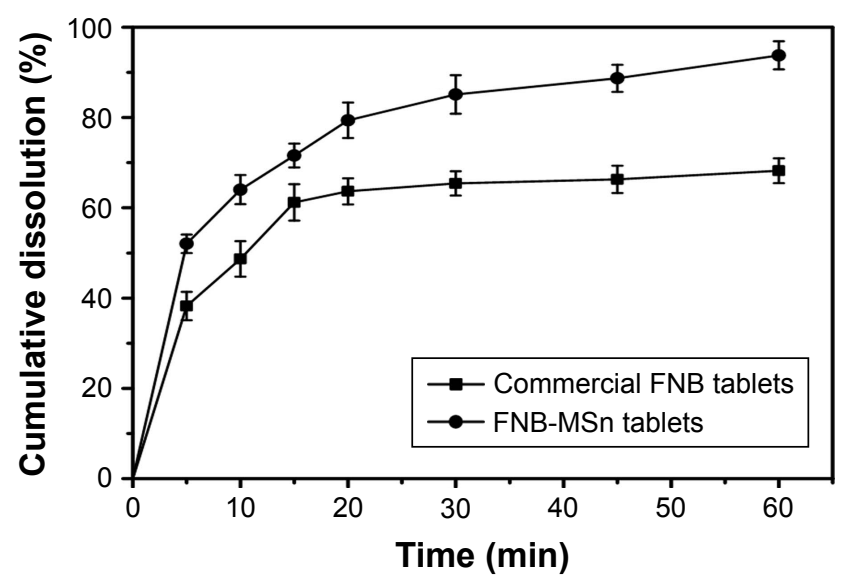

Figure 8 In vitro drug cumulative dissolution percentage patterns of FNB-MSn tablets and commercial FNB tablets.

Abbreviation: FNB, fenofibrate.

a significant enhancement compared with commercial FNB tablets. The cumulative dissolution rate of FNB-MSn tablets was over $93 \%$ in $1 \mathrm{~h}$, but only $\sim 68 \%$ of commercial FNB tablets had dissolved. In addition, the hardness of the prepared FNB-MSn tablets was up to $4.4 \pm 0.51 \mathrm{~kg} / \mathrm{cm}^{2}$. The friability was $0.87 \% \pm 0.06 \%$, and no cracks or fractures were observed in the FNB-MSn tablets. The disintegration time was $12 \mathrm{~min}$. The experimental results showed that the FNB-MSn tablets were in line with the tablet quality standard.

\section{In vivo pharmacokinetic study}

We prepared the FNB-MSn tablets and compared the in vivo release with commercial FNB tablets. The metabolites of FNB were the corresponding fenofibric acid. Therefore, fenofibric acid was measured to assess the in vivo pharmacokinetics of FNB. ${ }^{49}$ The FNB plasma concentration-time curves of the FNB-MSn tablets and commercial FNB tablets in rabbits are shown in Figure 9. The rate and extent of FNB absorption

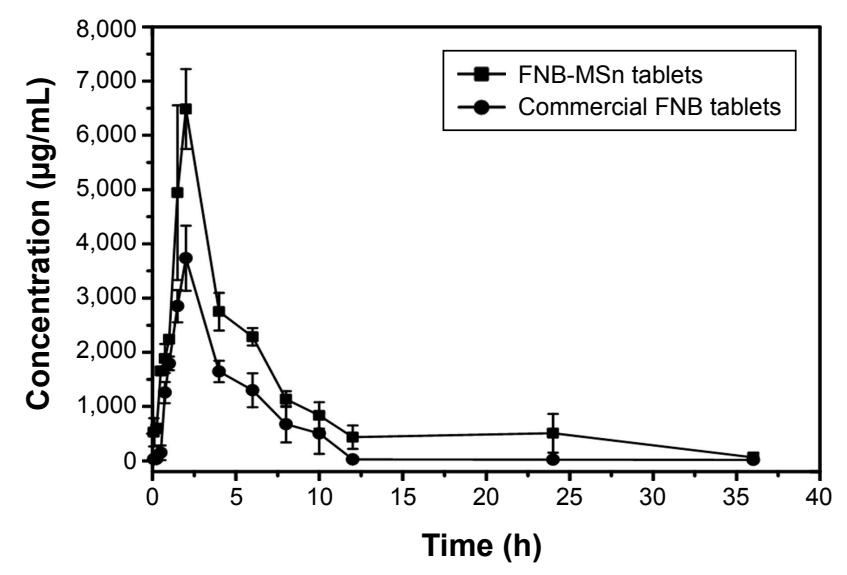

Figure 9 The FNB plasma concentration-time curves of FNB-MSn tablets and commercial FNB tablets in rabbits $(n=6)$.

Abbreviation: FNB, fenofibrate.
Table 3 Pharmacokinetic parameters of FNB after oral administration of FNB-MSn tablets and commercial FNB tablets

\begin{tabular}{lllll}
\hline Samples & $\begin{array}{l}\mathbf{C}_{\max } \\
(\mu \mathrm{g} / \mathbf{m L})\end{array}$ & $\mathbf{T}_{\max }(\mathbf{h})$ & $\mathbf{T}_{1 / 2}(\mathbf{h})$ & $\begin{array}{l}\mathbf{A U C}_{0-36} \\
(\mu \mathrm{g} / \mathbf{m L} \mathbf{h})\end{array}$ \\
\hline FNB-MSn tablets & $6,484.46$ & 2.00 & 6.83 & $35,979.93$ \\
Commercial FNB tablets & $3,734.26$ & 2.00 & 5.04 & $15,867.55$ \\
\hline
\end{tabular}

Notes: $\mathrm{AUC}_{0-36}$, area under the plasma concentration-time curve; $\mathrm{C}_{\text {max }}$, maximum plasma concentration; $T_{112}$, elimination half-life; $T_{\text {max }}$, time to reach $C_{\text {max }}$. Abbreviation: $\mathrm{FNB}$, fenofibrate.

for these FNB-MSn tablets were significantly higher than the rate and extent of FNB absorption for commercial FNB tablets, which was consistent with the results of the in vitro dissolution study. The pharmacokinetic parameters were calculated by the pharmacokinetic analysis software DAS 2.0, according to the non-compartmental method, and the results are provided in Table 3. The $\mathrm{C}_{\max }$ value $(p<0.05)$ and the $\mathrm{AUC}_{0-36}$ value $(p<0.05)$ of the prepared FNB-MSn tablets were up to $6,484.46 \mu \mathrm{g} / \mathrm{mL}$ and $35,979.93 \mu \mathrm{g} / \mathrm{mL} \cdot \mathrm{h}$, respectively. The $\mathrm{C}_{\max }$ value $(p<0.05)$ of the FNB-MSn tablets was much higher than the $\mathrm{C}_{\text {max }}$ value $(p<0.05)$ of the commercial FNB tablets, and the $\mathrm{AUC}_{0-36}$ value $(p<0.05)$ of the FNB-MSn tablets was more than twice as large as the $\mathrm{AUC}_{0-36}$ value $(p<0.05)$ of the commercial FNB tablets. The relative oral bioavailability of the FNB-MSn tablets was up to $226.75 \%$. The existing drug state of FNB-MSn resulted in significantly higher $\mathrm{C}_{\text {max }}$ and AUC for the drug than commercial FNB tablets. To the best of our knowledge, the amorphous state was the main reason for the rapid dissolution rate and high oral bioavailability. ${ }^{50}$ Amorphous drugs have a better dispersibility in comparison with crystalline drugs, which resulted in improved solubility and dissolution rate. The dissolved drugs were directly absorbed into the blood circulation. As a result, the relative oral bioavailability of FNB was effectively increased. These findings showed that MSn could significantly improve drug dissolution and enhance drug absorption.

\section{Conclusion}

In this study, MSn with mesoporous structure was successfully prepared for improving the dissolution and oral relative bioavailability of FNB. Its structure was determined by SEM and TEM. PXRD and DSC characterization demonstrated that FNB was incorporated into the mesoporous structure of MSn and was present in an amorphous state. The MTT assay revealed that MSn had better biocompatibility in Caco- 2 cells under the test conditions. In vitro drug dissolution studies showed that MSn greatly improved the dissolution rate of FNB. In the in vivo pharmacokinetic study, FNB-MSn tablets 
clearly improved the oral relative bioavailability of FNB in comparison with the commercial FNB tablets. These conclusions confirm the significant application potential of MSn as a novel carrier for poorly water-soluble drugs.

\section{Acknowledgment}

This study was supported by the National Natural Science Foundation of China (no 81302707), the Natural Science Foundation of Liaoning Province (no 20170540366), Liaoning province talent project support programs in colleges and universities (no LJQ2015065) and the Principal Fund-Aohong-Boze-Clinical Medicine Construction Special Fund (no XZJJ20140205).

\section{Disclosure}

The authors report no conflicts of interest in this work.

\section{References}

1. Lu M, Ho CT, Huang Q. Improving quercetin dissolution and bioaccessibility with reduced crystallite sizes through media milling technique. J Funct Foods. 2017;37:138-146.

2. de Mohac LM, de Fátima Pina M, Raimi-Abraham BT. Solid microcrystalline dispersion films as a new strategy to improve the dissolution rate of poorly water soluble drugs: a case study using olanzapine. Int J Pharm. 2016;508(1-2):42-50.

3. Salunkhe NH, Jadhav NR, More HN, Jadhav AD. Screening of drugsericin solid dispersions for improved solubility and dissolution. Int $J$ Boil Macromol. 2018;107(pt B):1683-1691.

4. Lai F, Pini E, Angioni G, et al. Nanocrystals as tool to improve piroxicam dissolution rate in novel orally disintegrating tablets. Eur J Pharm Biopharm. 2011;79(3):552-558.

5. Zhao P, Wang L, Sun C, et al. Uniform mesoporous carbon as a carrier for poorly water soluble drug and its cytotoxicity study. Eur J Pharm Biopharm. 2012;80(3):535-543.

6. Zhao $\mathrm{P}$, Jiang $\mathrm{H}$, Jiang $\mathrm{T}$, et al. Inclusion of celecoxib into fibrous ordered mesoporous carbon for enhanced oral bioavailability and reduced gastric irritancy. Eur J Pharm Sci. 2012;45(5):639-647.

7. Niu X, Wan L, Hou Z, et al. Mesoporous carbon as a novel drug carrier of fenofibrate for enhancement of the dissolution and oral bioavailability. Int J Pharm. 2013;452(1-2):382-389.

8. Zhu W, Zhao Q, Sun C, et al. Mesoporous carbon with spherical pores as a carrier for celecoxib with needle-like crystallinity: improve dissolution rate and bioavailability. Mater Sci Eng C Mater Biol Appl. 2014; 39:13-20.

9. Zhang Y, Zhi Z, Jiang T, Zhang J, Wang Z, Wang S. Spherical mesoporous silica nanoparticles for loading and release of the poorly watersoluble drug telmisartan. J Control Release. 2010;145(3):257-263.

10. Heikkilä T, Salonen J, Tuura J, et al. Mesoporous silica material TUD-1 as a drug delivery system. Int J Pharm. 2007;331(1):133-138.

11. Ambrogi V, Perioli L, Pagano C, et al. Use of SBA-15 for furosemide oral delivery enhancement. Eur J Pharm Sci. 2012;46(1-2):43-48.

12. Li-hong $\mathrm{W}$, Xin $\mathrm{C}$, Hui $\mathrm{X}$, et al. A novel strategy to design sustainedrelease poorly water-soluble drug mesoporous silica microparticles based on supercritical fluid technique. Int J Pharm. 2013;454(1):135-142.

13. He Y, Liang $\mathrm{S}$, Long $\mathrm{M}, \mathrm{Xu} \mathrm{H}$. Mesoporous silica nanoparticles as potential carriers for enhanced drug solubility of paclitaxel. Mater $S c i$ Eng C Mater Biol Appl. 2017;78:12-17.

14. Zhang Y, Wang H, Gao C, Li X, Li L. Highly ordered mesoporous carbon nanomatrix as a new approach to improve the oral absorption of the water-insoluble drug, simvastatin. Eur J Pharm Sci. 2013;49(5): 864-872.
15. Yang J, Alvebratt C, Zhang P, et al. Enhanced release of poorly watersoluble drugs from synergy between mesoporous magnesium carbonate and polymers. Int J Pharm. 2017;525(1):183-190.

16. Geszke-Moritz M, Moritz M. APTES-modified mesoporous silicas as the carriers for poorly water-soluble drug. Modeling of diflunisal adsorption and release. Appl Surf Sci. 2016;368:348-359.

17. Bakrudeen HB, Sugunalakshmi M, Reddy BSR. Auto-fluorescent mesoporous $\mathrm{ZnO}$ nanospheres for drug delivery carrier application. Mater Sci Eng C Mater Biol Appl. 2015;56:335-340.

18. Kapoor S, Hegde R, Bhattacharyya AJ. Influence of surface chemistry of mesoporous alumina with wide pore distribution on controlled drug release. J Control Release. 2009;140(1):34-39.

19. Xu W, Riikonen J, Lehto VP. Mesoporous systems for poorly soluble drugs. Int J Pharm. 2013;453(1):181-197.

20. Cao N, Li M, Zhao Y, et al. Fabrication of $\mathrm{SnO}_{2}$ /porous silica/polyethyleneimine nanoparticles for $\mathrm{pH}$-responsive drug delivery. Mater Sci Eng C Mater Biol Appl. 2016;59:319-323.

21. Surahman H, Krisnandi YK, Gunlazuardi J. Preparation and characterization of transparent conductive $\mathrm{SnO}_{2}-\mathrm{F}$ thin film deposited by spray pyrolysis: relationship between loading level and some physical properties. Procedia Environ Sci. 2015;28:242-251.

22. Rebholz J, Dee C, Weimar U, et al. A self-doping surface effect and its influence on the sensor performance of undoped $\mathrm{SnO}_{2}$ based gas sensors. Procedia Eng. 2015;120:83-87.

23. Kaçar C, Erden PE, Kılıç E. Amperometric 1-lysine biosensor based on carboxylated multiwalled carbon nanotubes- $\mathrm{SnO}_{2}$ nanoparticlesgraphene composite. Appl Surf Sci. 2017;419:916-923.

24. Okazaki T, Seino S, Nakagawa T, et al. Radiochemical synthesis of a carbon-supported $\mathrm{Pt}-\mathrm{SnO}_{2}$ bicomponent nanostructure exhibiting enhanced catalysis of ethanol oxidation. Radiat Phys Chem. 2015; 108:1-6.

25. Xiao H, Yao S, Qu F, et al. Electrochemical energy storage performance of heterostructured $\mathrm{SnO}_{2} @ \mathrm{MnO}_{2}$ nanoflakes. Ceram Int. 2017; 43:1688-1694.

26. Sumithra M, Rao PR, Nagaratnam A, et al. Characterization of $\mathrm{SnO}_{2}$ nanoparticles in the traditionally prepared ayurvedic medicine. Mater Today. 2015;2:4636-4639.

27. Sudhaparimala S, Vaishnavi M. Biological synthesis of nano composite $\mathrm{SnO}_{2}-\mathrm{ZnO}$ - screening for efficient photocatalytic degradation and antimicrobial activity. Mater Today. 2016;3:2373-2380.

28. Bukara K, Schueller L, Rosier J, et al. In vivo performance of fenofibrate formulated with ordered mesoporous silica versus 2 -marketed formulations: a comparative bioavailability study in beagle dogs. J Pharm Sci. 2016;105(8):2381-2385.

29. Liu YM, Xu J, He L, et al. Facile synthesis of fe-loaded mesoporous silica by a combined detemplation-incorporation process through Fenton's chemistry. J Phys Chem C. 2008;112:16575-16583.

30. Purwajanti $S$, Zhang $H$, Huang $X$, et al. Mesoporous magnesium oxide hollow spheres as superior arsenite adsorbent: synthesis and adsorption behavior. ACS Appl Mater Interfaces. 2016;8(38):25306-25312.

31. Gaudin P, Dorge S, Nouali H, et al. Synthesis of CuO/SBA-15 adsorbents for $\mathrm{SO}_{\mathrm{x}}$ removal applications, using different impregnation methods. C R Chim. 2015;18:1013-1029.

32. Xu J, Zhao Z, Hao Y, et al. Preparation of a novel form of gelatin with a three-dimensional ordered macroporous structure to regulate the release of poorly water-soluble drugs. J Pharm Sci. 2016;105(9):2940-2948.

33. Zhao Z, Gao Y, Wu C, Hao Y, Zhao Y, Xu J. Development of novel core-shell dual-mesoporous silica nanoparticles for the production of high bioavailable controlled-release fenofibrate tablets. Drug Dev Ind Pharm. 2016;42(2):199-208.

34. Niu X, Wan L, Hou Z, et al. Mesoporous carbon as a novel drug carrier of fenofibrate for enhancement of the dissolution and oral bioavailability. Int J Pharm. 2013;452(1-2):382-389.

35. Quan G, Wu Q, Zhang X, et al. Enhancing in vitro dissolution and in vivo bioavailability of fenofibrate by solid self-emulsifying matrix combined with SBA-15 mesoporous silica. Colloids Surf B Biointerfaces. 2016;141:476-482. 
36. Wan MM, Sun XD, Liu S, et al. Versatile drug releaser derived from the Ti-substituted mesoporous silica SBA-15. Microporous Mesoporous Mater. 2014;199:40-49.

37. Wu W, Ye C, Xiao H, et al. Hierarchical mesoporous silica nanoparticles for tailorable drug release. Int J Pharm. 2016;511(1):65-72.

38. Wu C, Zhao Z, Zhao Y, et al. Preparation of a push-pull osmotic pump of felodipine solubilized by mesoporous silica nanoparticles with a core-shell structure. Int J Pharm. 2014;475(1-2):298-305.

39. Ambrogi V, Perioli L, Pagano C, et al. Use of SBA-15 for furosemide oral delivery enhancement. J Pharm Sci. 2012;46(1-2):43-48.

40. Wang Z, Chen B, Quan G, et al. Increasing the oral bioavailability of poorly water-soluble carbamazepine using immediate-release pellets supported on SBA-15 mesoporous silica. Int J Nanomedicine. 2012; 7:5807.

41. Alexa IF, Ignat M, Popovici RF, Timpu D, Popovici E. In vitro controlled release of antihypertensive drugs intercalated into unmodified SBA-15 and MgO modified SBA-15 matrices. Int J Pharm. 2012; 436(1-2):111-119.

42. Kumar PN, Mary JSS, Chandrakala V, et al. Investigation of superior electro-optical properties of $\mathrm{SnO}_{2} / \mathrm{SiO}_{2}$ nanocomposite over its individual counterpart $\mathrm{SnO}_{2}$ nanoparticles. Mater Chem Phys. 2017;193:234-243.

43. Biswas N. Modified mesoporous silica nanoparticles for enhancing oral bioavailability and antihypertensive activity of poorly water soluble valsartan. Eur J Pharm Sci. 2017;99:152-160.

44. Jadhav NV, Vavia PR. Supercritical processed starch nanosponge as a carrier for enhancement of dissolution and pharmacological efficacy of fenofibrate. Int J Biol Macromol. 2017;99:713-720.
45. Wu C, Sun X, Zhao Z, et al. Synthesis of novel core-shell structured dual-mesoporous silica nanospheres and their application for enhancing the dissolution rate of poorly water-soluble drugs. Mater Sci Eng C Mater Biol Appl. 2014;44:262-267.

46. Nguyen THD, Vardhanabhuti B, Lin M, et al. Antibacterial properties of selenium nanoparticles and their toxicity to Caco-2 cells. Food Control. 2017;77:17-24.

47. Bedoya-RamírezD, Cilla A, Contreras-Calderón J, Alegría-Torán A. Evaluation of the antioxidant capacity, furan compounds and cytoprotective/ cytotoxic effects upon Caco-2 cells of commercial Colombian coffee. Food Chem. 2017;219:364-372.

48. Zhao Y, Wu C, Zhao Z, et al. Preparation of starch macrocellular foam for increasing the dissolution rate of poorly water-soluble drugs. Pharm Dev Technol. 2016;21(6):749-754.

49. Chen Y, Lu Y, Chen J, et al. Enhanced bioavailability of the poorly water-soluble drug fenofibrate by using liposomes containing a bile salt. Int J Pharm. 2009;376(1-2):153-160.

50. Hao Y, Wu C, Zhao Z, et al. Development of a novel starch with a three-dimensional ordered macroporous structure for improving the dissolution rate of felodipine. Mater Sci Eng C Mater Biol Appl. 2016; 58:1131-1137.
Drug Design, Development and Therapy

\section{Publish your work in this journal}

Drug Design, Development and Therapy is an international, peerreviewed open-access journal that spans the spectrum of drug design and development through to clinical applications. Clinical outcomes, patient safety, and programs for the development and effective, safe, and sustained use of medicines are the features of the journal, which

\section{Dovepress}

has also been accepted for indexing on PubMed Central. The manuscript management system is completely online and includes a very quick and fair peer-review system, which is all easy to use. Visit http://www.dovepress.com/testimonials.php to read real quotes from published authors. 